Research Article

\title{
Enterprise Credit Risk Management Using Multicriteria Decision-Making
}

\author{
Wenjuan Liu \\ Finance Department, Henan Vocational and Technical College, Zhengzhou, Henan450046, China \\ Correspondence should be addressed to Wenjuan Liu; liuwenjuan2021@126.com
}

Received 8 August 2021; Revised 16 September 2021; Accepted 27 September 2021; Published 24 November 2021

Academic Editor: Zaoli Yang

Copyright ( $(2021$ Wenjuan Liu. This is an open access article distributed under the Creative Commons Attribution License, which permits unrestricted use, distribution, and reproduction in any medium, provided the original work is properly cited.

\begin{abstract}
The purpose of this study is to reduce the rate of multicriteria decision-making (MCDA) errors in credit risk management and to weaken the influence of different attitudes of enterprise managers on the final decision when facing credit risk. First, several solutions that are suitable for present enterprise credit risk management are proposed according to the research of enterprise risk management in the world. Moreover, the criteria and matrix are established according to the general practice of the expert method. A decision-making method of enterprise credit risk management with trapezoidal fuzzy number as the criteria of credit risk management is proposed based on the prospect theory; then, the weight is calculated based on $G_{1}$ weight calculation, $G_{2}$ weight calculation method, and the method of maximizing deviation; finally, the prospect values of the alternatives calculated by each method are adopted to sort and compare the proposed solutions. Considering the difference of risk degree of managers in the face of credit risk management, the ranking results of enterprise credit risk management solutions based on three weight calculation methods are compared. The results show that as long as the quantitative value of the risk attitude of the enterprise credit risk manager meets a certain range, the final choice of credit risk management scheme ranking is consistent. This exploration provides a new research direction for enterprise credit risk management, which has reference significance.
\end{abstract}

\section{Introduction}

The credit risk management system of all kinds of enterprises in China is not perfect in the context of the development of Big Data Internet and deeper opening to the outside world, and the awareness of risk management is insufficient. Risk managers often make mistakes in risk management decisions in Internet finance, supply chain finance, and another convenient virtual trading environment with credit risk, leading to the enterprise into a strategic or financial crisis. Credit risk is particularly serious in small- and medium-sized enterprises. Small- and medium-sized enterprises have prominent problems in credit risk management due to the lack of understanding of credit risk management, which seriously restricts the development of enterprises. To solve the problems of credit risk management and respond to the needs of the construction and development of the national social credit system, many scholars have studied the related problems.
Many scholars have put forward relevant countermeasures for the credit management risk of enterprises in a certain industry based on the actual situation of enterprises in this field. Li put forward suggestions from the aspects of receivables, customer credit investigation, and risk early warning culture [1]; Wang observed and discussed the modernization of national governance capacity from the perspective of enterprise credit risk management. In fact, enterprise credit risk management exerts a profound impact on the modernization of China's national governance capacity. In this regard, the construction of risk management system standardization is very critical [2]. Xiao preliminarily analyzed the application prospect of financial products in enterprise credit risk management based on the characteristics of common financial products and gave relevant application suggestions [3]. The above research is based on the industry to make recommendations on the credit risk management of related companies, but the weight of these recommendations and the priority of the 
program are not studied. The research method based on multicriteria decision-making (MCDA) proposed has been studied by some scholars. Han et al. applied the method of decision-making and evaluation laboratory to analyze the interaction among decision-making criteria and obtained the key comprehensive weight in the case of relying on criteria. The reference point is the average alternative state value. The comprehensive prospect value under the risk state of the alternative is solved as the decision basis according to the prospect theory [4]. Zhang et al. used MCDA theory and data mining technology and proposed a new method for credit risk identification, evaluation, and management [5]. Ma et al. proposed a credit evaluation model based on multimodel combination algorithm. The weighted voting combination was carried out on three stable classifiers, random forest, weak classifier (decision tree), and support vector machine. In feature extraction, an improved feature extraction method was adopted. First, the importance of personal credit data features was analyzed according to Fisher's ratio, and the features were divided according to different values. In the training model, the improved grid search algorithm was used to optimize the parameters of the model to improve the optimization speed and efficiency. Finally, they concluded that the performance of weak classifier (decision tree) model is better than random forest and support vector machine, and the performance of combined model is better than that of single model [6]. According to the opinions of experts, Mohammadfam used the fuzzy decision laboratory analysis (DEMATEL) method to determine the cause-effect relationships and the interaction of the influential factors affecting accident occurrence and determine the key root factors. They found that "organizational safety attitude," "safety communication," "work and safety training," and "system safety design" were the root cause variables. It was concluded that organizational factors and some individual variables are the key factors affecting the occurrence of accidents, and the corrective measures for accident prevention should mainly focus on correcting these variables [7]. Although they used MCDA theory in their research, there are few research decisions in the combination of enterprise credit risk management.

Further demonstration research is made based on the research of fuzzy MCDA based on prospect theory [8], and this exploration expands to the field of enterprise credit risk management. The first part is the research background, the current research on enterprise credit risk management in the world, as well as the research framework and research contribution. The second part is mainly to introduce the relevant theories and methods, as well as the determination steps of the scheme and criteria. The third part is mainly to introduce the result data of the experiment. According to the theoretical method of the second part, the results of weight calculation and scheme ranking are discussed and analyzed. The fourth part is the conclusion of this exploration. Finally, there are references and so on. According to the literature and expert method, the implementation scheme and criteria of enterprise credit risk management are formulated in detail, and the weights of MCDA schemes are verified and calculated by different methods. Finally, the ranking of the best schemes of enterprise credit risk management is obtained. The research innovation is that it introduces the value of decision-maker's subjective attitude $\lambda$, and the influence of decision-maker's subjective factors can be minimized by adjusting this value. In the current complex economic environment, this exploration has guiding significance for the enterprise's own credit risk management. It is also a reference for the third-party capital market with enterprise credit risk as the enterprise assessment index, and a positive promotion for the economic supervision department to prevent systematic credit risk.

\section{Related Methods and Theories}

2.1. MCDA Methods. One of the main modules of decision analysis theory is MCDA, and the selection of the best scheme or the comparison and optimization of multiple schemes is its main research direction. MCDA model is widely employed in engineering, economy, environment, national defense, and other fields. MCDA analysis is also called multiattribute decision analysis, multicriteria decision analysis, and multi-index decision analysis [9-12]. This model is an extension of the application of multialternative decision-making theory. Many algorithms of the MCDA model are summarized into three categories: scoring method, priority method, goal programming, and reference point model. As the name suggests, the scoring method is to make decisions by calculating the score of each scheme; the priority method is to group the schemes, then compare them, and make decisions according to the order; the mechanism of the third kind of method is to select the scheme which is close to the original set possible value from multiple schemes for decision-making [13-16]. Figure 1 displays the process of MCDA implementation.

\subsection{Prospect Theory. Prospect theory was proposed by} Daniel Kahneman and Amos Tversky. It applies psychological research to economics and makes outstanding contributions to human judgment and decision-making under uncertainty. In view of the rational man hypothesis, which has been used for a long time, the prospect theory reveals the irrational psychological factors affecting choice behavior from the psychological and behavioral characteristics of people. In prospect theory, there are mainly two kinds: decision weight $\pi$ and value $v$ function. It is essential to use the $t$ function created by Kahneman and Tversky to clearly measure the characteristics that decision-makers do not pay attention to risk when they see profit and care about risk when they see loss. The decision factor when decisionmakers see profit is represented by $\pi^{+} . \pi^{-}$represents the amount of decision factor in the face of loss [17].

\subsection{Fuzzy Set Theory. The purpose of the research of human} system and social system is to use fuzzy system theory to solve the issues that are full of uncertainty, including complex behavior of people, psychological analysis, the change trend of social economy, the model, prediction, and decision support of various social phenomena. This aspect includes the 


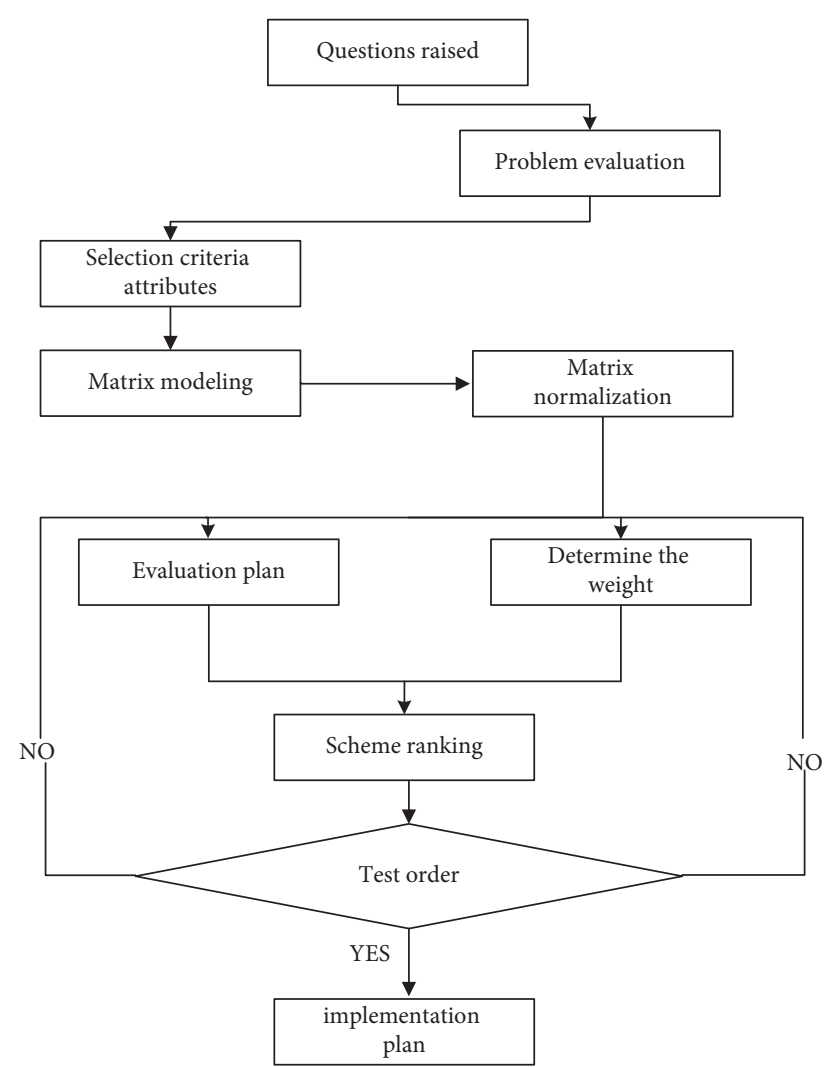

Figure 1: Schematic diagram of MCDA implementation process.

prediction and complete evaluation of various crises, the evaluation method of human error system, the establishment of the model of bad structure system, the application of fuzzy theory in system fault detection and diagnosis, human behavior and psychological analysis. The basis of fuzzy system theory is fuzzy set theory. The methods of fuzzy system theory are mainly adopted in environmental quality evaluation, such as fuzzy clustering method and fuzzy comprehensive evaluation method. Its core is to use membership degree to describe massive fuzzy boundaries in objective things. In environmental quality assessment, the attribution of assessment level, that is, the relationship between elements and sets, is no longer the belonging or nonbelonging relationship in the general classical set theory, but a number in the middle of [0, 1], which can more accurately reflect the actual situation. If the property of the fuzzy number $\widetilde{A}$ in a fuzzy set is a membership function which is bounded on a real line $R$ and has normality, continuity and fuzzy convexity, the $\alpha$-level set of $\widetilde{A}$ is set to $\widetilde{A}^{\alpha}=\left[\widetilde{A}_{L}^{\alpha}, \widetilde{A}_{R}^{\alpha}\right](\alpha>0)[18]$.

Definition 1. Distance between two fuzzy numbers $\widetilde{A}$ and $\widetilde{B}$ is

$$
d(\widetilde{A}, \widetilde{B}) \int_{0}^{1}\left[(1-\lambda)\left(\widetilde{A}_{L}^{\alpha}-\widetilde{B}_{L}^{\alpha}\right)+\lambda\left(\widetilde{A}_{R}^{\alpha}-\widetilde{B}_{R}^{\alpha}\right)\right] \mathrm{d} \alpha
$$

$\lambda \in[0,1]$ represents the value of the risk attitude of managers. When $\lambda>0.5$, managers tend to consider risks; when $\lambda=0.5$, the decision-maker is risk-neutral; when $\lambda<0.5$, managers avoid considering risks $[19,20]$.
For trapezoidal fuzzy numbers $\widetilde{A}=\left[a_{1}, a_{2}, a_{3}, a_{4}\right]$ and $\widetilde{B}=\left[b_{1}, b_{2}, b_{3}, b_{4}\right]$, there is

$$
\begin{aligned}
d_{\lambda}(\widetilde{A}, \widetilde{B})= & \frac{1}{2}\left\{\left[\left(a_{2}-b_{2}\right)+\left(a_{1}-b_{1}\right)\right](1-\lambda)\right. \\
& \left.+\lambda\left[\left(a_{3}-b_{3}\right)+\left(a_{4}-b_{4}\right)\right]\right\} .
\end{aligned}
$$

2.4. Risk-Based MCDA Process Based on Prospect Theory. There is the MCDA problem under a certain risk state. It is set that the scheme set is $A=\left\{a_{1}, a_{2}, \ldots, a_{n}\right\}$ and the criterion set is $C=\left\{c_{1}, c_{2}, \ldots, c_{m}\right\}$. Each criterion is not related to each other, and the corresponding weight vector is $\omega=\left(\omega_{1}, \omega_{2}, \ldots, \omega_{m}\right)^{T}$. The trapezoidal fuzzy number $\tilde{a}_{i j}\left(\tilde{a}_{i j}=\left[\tilde{a}_{i j}^{1}, \tilde{a}_{i j}^{2}, \tilde{a}_{i j}^{3}, \tilde{a}_{i j}^{4}\right]\right)$ is the value of the scheme $a_{i}$ in criterion $c_{j}$. The weight information of all criteria is different. The decision-making process of risk-based fuzzy decisionmaking problem is described in detail as follows:

The first step is to standardize the decision information.

The decision matrix $D=\left(\tilde{a}_{i j}\right)_{n \times m}$ consists of the value $\widetilde{a}_{i j}$ of the scheme $a_{i}$ in criterion $c_{j}$. The interference of different factors on decision-making can be eliminated by normalizing the decision-making matrix $D$. The decision matrix $R=\left(\widetilde{Y}_{i j}\right)_{n \times m}$ is set to the normalized $\tilde{\Upsilon}_{i j}=\left[\tilde{\Upsilon}_{i j}^{1}, \tilde{\Upsilon}_{i j}^{2}, \tilde{\Upsilon}_{i j}^{3}, \tilde{\Upsilon}_{i j}^{4}\right]$.

For the cost-based criteria, there are

$$
\mathscr{A}_{i j}^{k}=\frac{\max _{i}\left(a_{i j}^{4}\right)-a_{i j}^{k}}{\max _{i}\left(a_{i j}^{4}\right)-\min _{i}\left(a_{i j}^{1}\right)}, \quad k=1,2,3,4 .
$$

For the profit-making criteria, there are

$$
\mathscr{A}_{i j}^{k}=\frac{a_{i j}^{k}-\min _{i}\left(a_{i j}^{1}\right)}{\max _{i}\left(a_{i j}^{4}\right)-\min _{i}\left(a_{i j}^{1}\right)}, \quad k=1,2,3,4 .
$$

The second step is to select a reference point.

The profit or loss is measured by decision-makers according to the reference point, and the decisionmaker makes a decision after calculating the prospect value of the scheme. Therefore, the key to decisionmaker behavior is the choice of reference point. The reference points here are selected as positive and negative ideal points, $G_{k}^{+}=\max _{1 \leq i \leq n}\left(\widetilde{\Upsilon}_{i k}\right)$ and $G_{k}^{-}=\min _{1 \leq i \leq n}\left(\widetilde{\Upsilon}_{i k}\right)$, respectively.

The third step is to calculate the value function of the scheme criteria.

Equation (1) suggests that the value function of the scheme $a_{i}$ in the criterion $c_{j}$ is

$$
v\left(\widetilde{\Upsilon}_{i j}\right)=\left\{\left(d\left(\widetilde{\Upsilon}_{i j}, G_{j}^{-}\right)\right)^{\alpha}-\theta\left(d\left(\widetilde{\Upsilon}_{i j}, G_{j}^{+}\right)\right)^{\beta} .\right.
$$

In equation (5), $G_{j}^{-}$is the negative ideal scheme and $G_{j}^{+}$ is the positive ideal scheme. Equation (2) reveals that the distance set of the scheme $a_{i}$ from criterion $c_{j}$ to positive and negative ideal points is 


$$
\begin{aligned}
& d\left(a_{i}, G^{+}\right)=\left\{d\left(\widetilde{\Upsilon}_{i 1}, G_{1}^{+}\right), d\left(\widetilde{\Upsilon}_{i 2}, G_{2}^{+}\right), \ldots, d\left(\widetilde{\Upsilon}_{i m}, G_{m}^{+}\right)\right\}, \\
& d\left(a_{i}, G^{-}\right)=\left\{d\left(\widetilde{\Upsilon}_{i 1}, G_{1}^{-}\right), d\left(\widetilde{\Upsilon}_{i 2}, G_{2}^{-}\right), \ldots, d\left(\widetilde{\Upsilon}_{i m}, G_{m}^{-}\right)\right\} .
\end{aligned}
$$

The fourth step is to calculate the comprehensive prospect value of each scheme.

The sum of the positive prospect $V^{+}$and the negative prospect $V^{-}$is the criterion $c_{j}$. The comprehensive prospect value of the scheme $a_{i}$ is

$$
V_{i}=\sum_{j=1}^{m} v_{i j}^{+} \pi^{+}\left(\omega_{j}\right)+\sum_{j=1}^{m} v_{i j}^{-} \pi^{-}\left(\omega_{j}\right)
$$

The fifth step is to determine the weight of the criteria and the order of the schemes.

$G_{1}$ method is adopted to determine weight [21].

The first step is to determine the order of the criteria from large to small

The second step is the ideal assignment of the importance ratio $\gamma_{j}\left(\gamma_{j}=c_{j-1} / c_{j}\right)$ between the criteria $c_{j-1}$ and $c_{j}$ specified by experts

The third step is to calculate the weight $\omega_{k}$ of the $k$ th criterion is

$$
\omega_{k}=\left(1+\sum_{j=2}^{k} \prod r j\right)^{-1} .
$$

The fourth step is to know the weight expressions of criteria $k-1, \ldots, 3$ and 2 from weight $\omega_{k}$.

$$
\omega_{j-1}=r_{j} \omega_{j} .
$$

$G_{2}$ method is adopted to determine weight [22].

The first step is to determine the order relationship among the criteria

The second step is that the experts specify the most unimportant criterion $c_{k}$; the third step is that the experts give the optimal value of $c_{k}$, which is the ratio of the importance degree of other criteria $c_{j}$ and $c_{k}$; the fourth step is the weight $\omega_{k}$ of the criterion $k$ :

$$
\omega_{k}=\frac{d_{k}}{\sum_{k=1}^{m} d_{k}} .
$$
[23].

Maximizing deviation is employed to determine weights

The algorithm to reflect the importance of criterion by calculating the proportion of the deviation of criterion $j$ to the total deviation of all criteria is the maximizing deviation method. The larger the proportion is, the greater the corresponding weight is. $t_{i j}(i=1,2, \ldots, n ; j=1,2, \ldots, m)$ is set as the $i$ th scheme. After normalization of the $j$ th criterion, $\omega_{j}$ is the weight of the $j$ th criterion, and

$$
\omega_{j}=\frac{\sum_{i=1}^{n} \sum_{k=1}^{n}\left|t_{i j}-t_{k i}\right|}{\sum_{j=1}^{m} \sum_{i=1}^{n} \sum_{k=1}^{n}\left|t_{i j}-t_{k i}\right|} .
$$

2.5. Credit Risk Management Theory. Credit risk can be defined as follows in the current environment. The debtor's credit rating is affected by various factors, and asset value has changed to a certain extent, which may bring certain losses to the debtor. Enterprise credit risk management mainly includes the following aspects. In the early stage of contract signing and credit risk management, the capital credit information of the other party is collected and evaluated; in the middle stage of contract signing and credit risk management, a guarantee mechanism of creditor's rights should be established; in the late stage of contract signing and credit risk management, the mechanism of receivables recovery and management should be established. Generally, enterprise credit risk management includes risk identification, risk assessment, risk decision-making, and risk management evaluation. Through the establishment of credit risk management mechanism, it can effectively evaluate customer information, increase customer credit limit, and strengthen the protection of creditor's rights. It is conducive to coordinating the internal work of the enterprise, promoting the exchange and communication among various departments, and improving the security and stability of contract implementation, account recovery, and other work contents [24]. Figure 2 presents the organizational structure of enterprise credit risk management.

2.6. Establishment of Enterprise Credit Risk Management Scheme and Criterion. SinRating summarizes the most obvious four negative problems in the management of credit risk through the investigation and credit rating of more than 30 business association enterprises every year, as well as through close communication with tens of thousands of insured households and consulting and cooperation of dozens of enterprises. The first negative problem is attitude. It means that attention is paid to the sales instead of risks. The second negative problem is that it is not comprehensive. Attention is paid to beginning but not ending. The third negative problem is science. There are no tools and methods. The fourth negative problem is profession. Professional organization and professional ideas are lacking. Experts from the American Management Association believe that ignorance in credit risk management will become one of the biggest obstacles to the development of new market environment [25].

Through the investigation and research on the credit risk management of enterprises in China, the problems of credit risk management in China enterprises, especially small- and medium-sized enterprises, are analyzed by means of literature method, and four schemes that can be used as decision-making of credit risk management are put forward. 


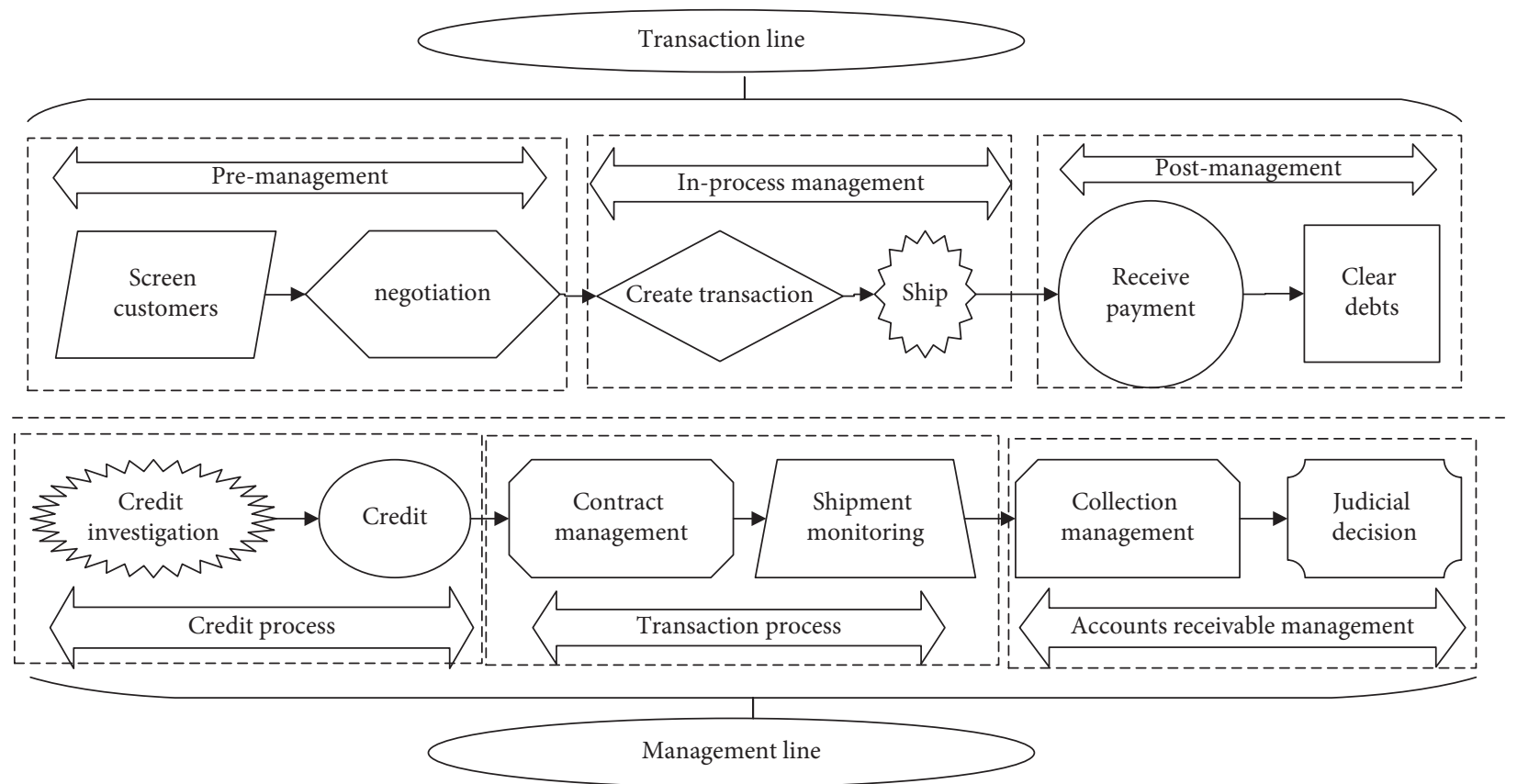

FIGURE 2: Organizational structure of enterprise credit risk management.

Scheme 1: At the national level, a dynamic Internet credit system platform based on Big Data should be established, the enterprise credit files and credit evaluation indicators should be improved, market access standards should be defined, the self-discipline behavior of the industry should be strengthened from the government level, and the enterprise credit reward and punishment mechanism should be established [26].

Scheme 2: the enterprise itself should improve its legal and risk awareness, create an efficient enterprise credit and risk management evaluation organization, improve the credit risk management system, cultivate a healthy risk culture, enhance the risk early warning mechanism, cultivate credit risk management professionals, strengthen the quality management of internal personnel, and strengthen the communication and cooperation among various departments.

Scheme 3: It is essential to strengthen the cash flow control, innovate the management methods, and strengthen the management mode of receivables [27].

Scheme 4: It is essential to improve the awareness of avoiding credit risk, establish an effective risk transfer mechanism, and transfer risks by means of creditor's rights, equity, and property rights.

Six benefit criteria for decision-making are formulated according to the characteristics of enterprise credit risk management, which are legitimacy, controllability, minimum negative effect, minimum cost, maximum profit, and minimum marginal cost.

\section{Research Results of Enterprise Credit Risk Management Scheme under MCDA}

The schemes 1 to 4 are numbered as $a_{1} \sim a_{4}$ according to the previous scheme, the criteria are numbered as $c_{1} \sim c_{6}$, and the criteria are all benefit criteria. The original values of each criterion need to be standardized to eliminate the interference of different factors on the decision-making results.

The standardized value of criterion $j$ in the scheme $i$ is $\tilde{\Upsilon}_{i j}$. Tables 1 and 2 display the results.

According to Definition 1, positive and negative ideal schemes are $G^{+}=\left\{\widetilde{\Upsilon}_{43}, \widetilde{\Upsilon}_{26}, \widetilde{\Upsilon}_{31}, \widetilde{\Upsilon}_{14}, \widetilde{\Upsilon}_{45}, \widetilde{\Upsilon}_{12}\right\} \quad$ and $G^{-}=\left\{\widetilde{Y}_{13}, \widetilde{\Upsilon}_{36}, \widetilde{Y}_{23}, \widetilde{\Upsilon}_{24}, \widetilde{\Upsilon}_{35}, \widetilde{\Upsilon}_{32}\right\}$, respectively.

The distance set from each scheme to positive and negative ideal schemes can be obtained according to equation (2). When the reference point is the positive ideal scheme, for the positive ideal scheme, scheme $a_{i}$ is worse, and the decision-maker is in the state of loss consciousness. The decision-maker focuses on the risk, $\lambda>0.5$, so $\lambda=0.8$ in the distance equation is taken; when the reference point is the negative ideal scheme, for the negative ideal scheme, scheme $a_{i}$ is better, and the decision-maker is in the state of income consciousness. At this time, the decision-maker is risk averse, $\lambda<0.5$, so the value $\lambda=0.3$ in the distance equation is taken.

The value function of each scheme to each criterion is calculated according to equation (5). Finally, the comprehensive prospect value of each scheme to each criterion is obtained according to equation (7). 
TABLE 1: Criterion values of each scheme after standardization under $c 1$ to $c 3$ criterion.

\begin{tabular}{ccccc}
\hline$a$ & $a_{1}$ & $a_{2}$ & $a_{3}$ & $a_{4}$ \\
\hline$c_{1}$ & {$[0.1,0.2,0.4,0.6]$} & {$[0.15,0.25,0.35,0.5]$} & {$[0.3,0.6,0.7,0.8]$} & {$[0.5,0.7,0.9,1.0]$} \\
$c_{2}$ & {$[0.4,0.6,0.8,0.9]$} & {$[0.3,0.4,0.6,0.7]$} & {$[0.2,0.3,0.4,0.6]$} & {$[0,0.3,0.4,0.5]$} \\
$c_{3}$ & {$[0.5,0.6,0.9,1.0]$} & {$[0,0.1,0.2,0.4]$} & {$[0.5,0.6,0.8,1.0]$} & {$[0.4,0.6,0.8,0.9]$} \\
\hline
\end{tabular}

TABLE 2: Criterion values of each scheme after standardization under $c 4$ to $c 6$ criterion.

\begin{tabular}{ccccc}
\hline$a$ & $a_{1}$ & $a_{2}$ & $a_{3}$ & $a_{4}$ \\
\hline$c_{4}$ & {$[0.3,0.5,0.7,0.9]$} & {$[0.35,0.45,0.55,0.55]$} & {$[0.2,0.4,0.6,0.7]$} & {$[0.1,0.3,0.4,0.6]$} \\
$c_{5}$ & {$[0.3,0.4,0.6,0.8]$} & {$[0.1,0.3,0.6,0.8]$} & {$[0,0.2,0.4,0.6]$} & {$[0.3,0.5,0.6,0.9]$} \\
$c_{6}$ & {$[0.4,0.7,0.8,1.0]$} & {$[0.5,0.6,0.8,1]$} & {$[0.4,0.5,0.6,0.75]$} & {$[0.3,0.6,0.8,0.9]$} \\
\hline
\end{tabular}

\subsection{Weight Determination by $G_{1}$}

(1) According to experts' opinions, the order of subjective influence among the six criteria is determined as $c_{1}>c_{2}>c_{3}>c_{4}>c_{5}>c_{6}$;

(2) The ideal values of the importance ratio $\gamma_{j}$ between criteria $c_{j-1}$ and $c_{j}$ are $\gamma_{2}=\left(c_{1} / c_{2}\right)=1.3, \quad \gamma_{3}=$ $\left(c_{2} / c_{3}\right)=1.3, \gamma_{4}=\left(c_{3} / c_{4}\right)=1.2, \gamma_{5}=\left(c_{4} / c_{5}\right)=1.1$, and $\gamma_{6}=\left(c_{5} / c_{6}\right)=1.2$;

(3) Substituting $\gamma_{j}$ into equations (9) and (10) can get the weights as follows: $\omega_{1}=0.2715, \omega_{2}=0.1925, \omega_{3}=$ $0.1641, \omega_{4}=0.1303, \omega_{5}=0.1211$, and $\omega_{6}=0.1146$.

\subsection{Weight Determination by $G_{2}$}

(1) Experts specify the least important criterion $c_{2}$;

(2) The experts give the following ideal value of the ratio of the importance degree of other criteria $c_{j}$ to $c_{2}$, which are $d_{1}=\left(c_{1} / c_{2}\right)=1.4, d_{2}=\left(c_{2} / c_{2}\right)=1$, $d_{3}=\left(c_{3} / c_{2}\right)=1.3, d_{4}=\left(c_{4} / c_{2}\right)=1.2, d_{5}=\left(c_{5} / c_{2}\right)$ $=1.2$, and $d_{6}=\left(c_{6} / c_{2}\right)=1.1$;

(3) The weights obtained by substituting $d_{j}$ into equation (12)are $\omega_{1}=0.1914, \omega_{2}=0.1627, \omega_{3}=0.1824$, $\omega_{4}=0.1349, \omega_{5}=0.1627$, and $\omega_{6}=0.1548$.

3.3. Weight Determination by Maximizing Deviation. Since $d_{\lambda}\left(\tilde{\gamma}_{i j}, \tilde{\gamma}_{k j}\right)=\left|\tilde{\gamma}_{i j}-\tilde{\gamma}_{k j}\right|$, according to the distance equation (2), $\lambda=0.5$ is taken and substituted into equation (12). The weights obtained are as follows: $\omega_{1}=0.1716$, $\omega_{2}=0.1962, \omega_{3}=0.1702, \omega_{4}=0.1625, \omega_{5}=0.1441$, and $\omega_{6}=0.1454$. Figure 3 presents the comprehensive prospect values calculated by the three methods.

Figure 3 displays that although the proportions determined by the three methods are different, the final order is the same, that is, $a_{1}>a_{4}>a_{3}>a_{2}$.

3.3.1. Study on $\lambda$ Value. In the case of loss, decision-makers tend to consider risk; in the face of income, decision-makers avoid considering risk. Whether the scheme selection changes with different values of $\lambda$ is discussed by adjusting the value of $\lambda$ in the distance equation. When the reference point is a positive ideal scheme, different values $(\lambda=0.6,0.7,0.9,1)$ are taken; when the reference point is a

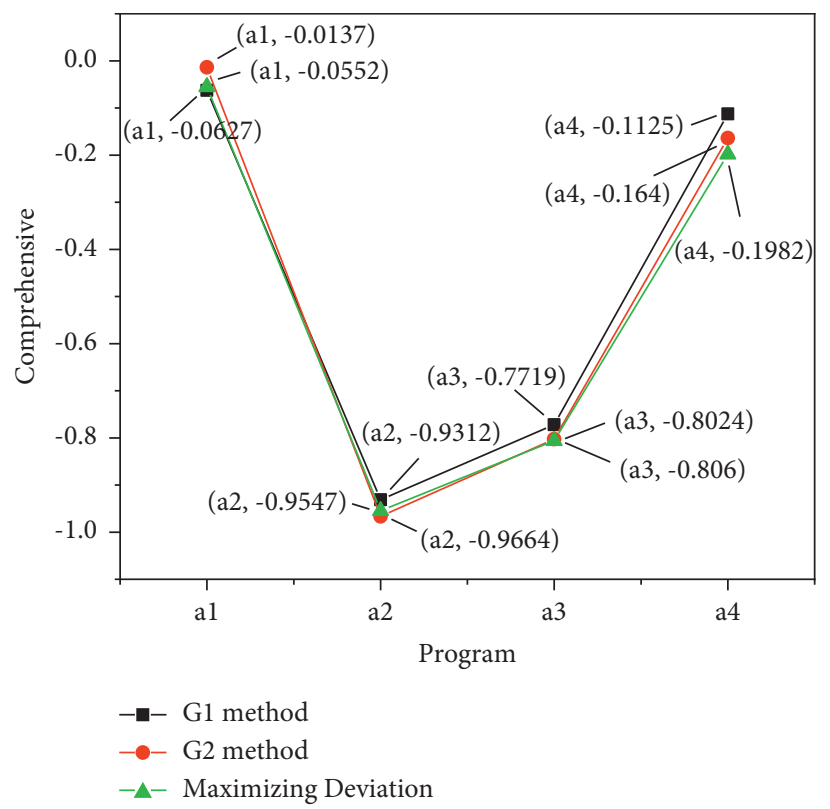

FIGURE 3: Schematic diagram of comprehensive prospect value and scheme sorting results.

negative ideal scheme, $\lambda=0.3$ is taken without changing. Figures 4 to 7 present the comprehensive prospect value calculated by the above method.

The above figures show that the value of $\lambda$ is taken as $(\lambda=0.6,0.7,0.9,1)$ when the reference point is a positive ideal scheme, and the value of $\lambda$ is taken as a fixed value $(\lambda=0.3)$ when the reference point is a negative ideal scheme. Although the comprehensive prospect values obtained by the three methods are different, the final scheme selection is the same, that is, $a_{1}>a_{4}>a_{3}>a_{2}$.

Similarly, $\lambda=0.8$ is taken without changing when the positive ideal scheme is taken as the reference point; different $\lambda$ values $(\lambda=0.6,0.7,0.9,1)$ are taken when the negative ideal scheme is taken as the reference point. The value of $\lambda(\lambda=0.8)$ is fixed when the positive ideal scheme is taken as the reference point. Different values of $\lambda$ $(\lambda=0,0.1,0.2,0.4)$ are taken when the negative ideal scheme is taken as the reference point. Although the comprehensive prospect values obtained from the above methods are different, the final scheme selection is the same, that is, $a_{1}>a_{4}>a_{3}>a_{2}$. 


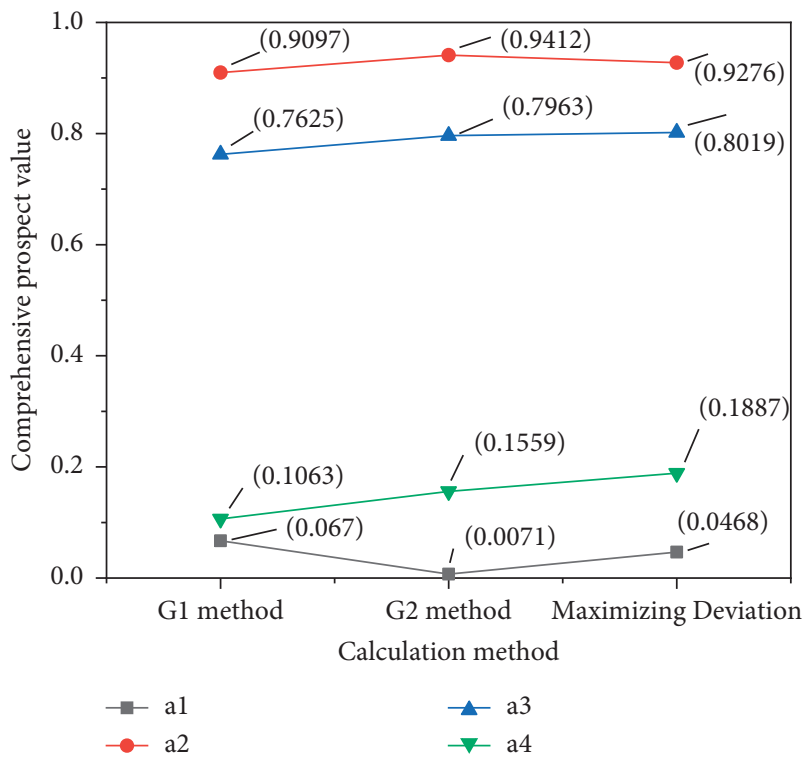

FIGURE 4: Schematic diagram of comprehensive prospect value and ranking results of schemes when $\lambda=0.6$.

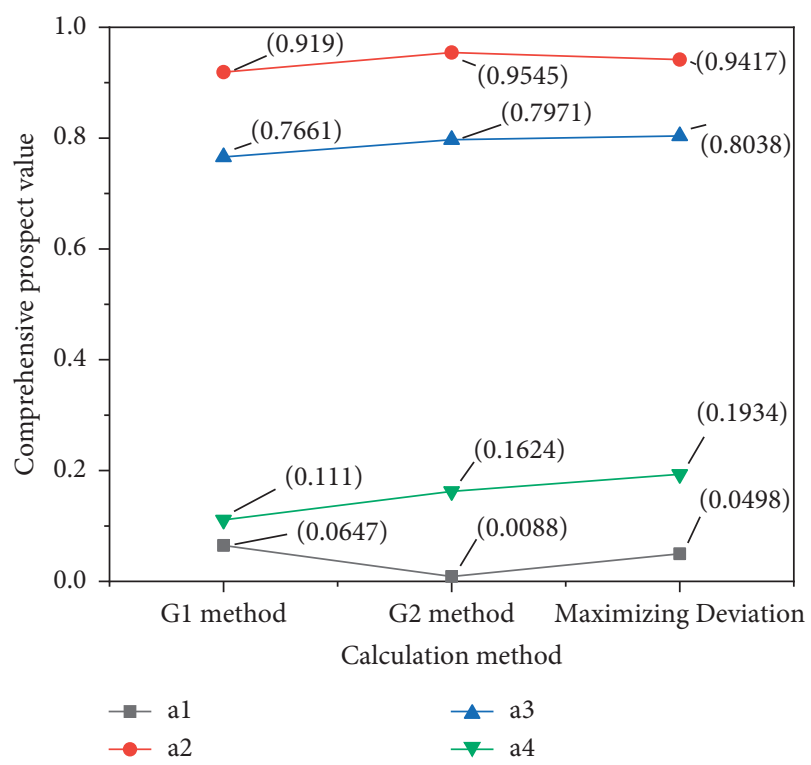

FIGURE 5: Schematic diagram of comprehensive prospect value and ranking results of schemes when $\lambda=0.7$.

The reference points are positive and negative ideal scheme and negative ideal scheme, and the value $\lambda$ changes at the same time. Figures 8 to 11 display the results.

Figure 8 displays that $\lambda=0.6$ is taken if the reference point is positive and ideal; $\lambda=0.1$ is taken when the reference point is a negative ideal scheme. Figure 8 presents the calculation results of the comprehensive prospect value of each method, and the ranking of the final scheme remains unchanged, which is $a_{1}>a_{4}>$ $a_{3}>a_{2}$. In Figures 9 to 11 , when the reference point is the positive or negative ideal scheme, no matter how the value

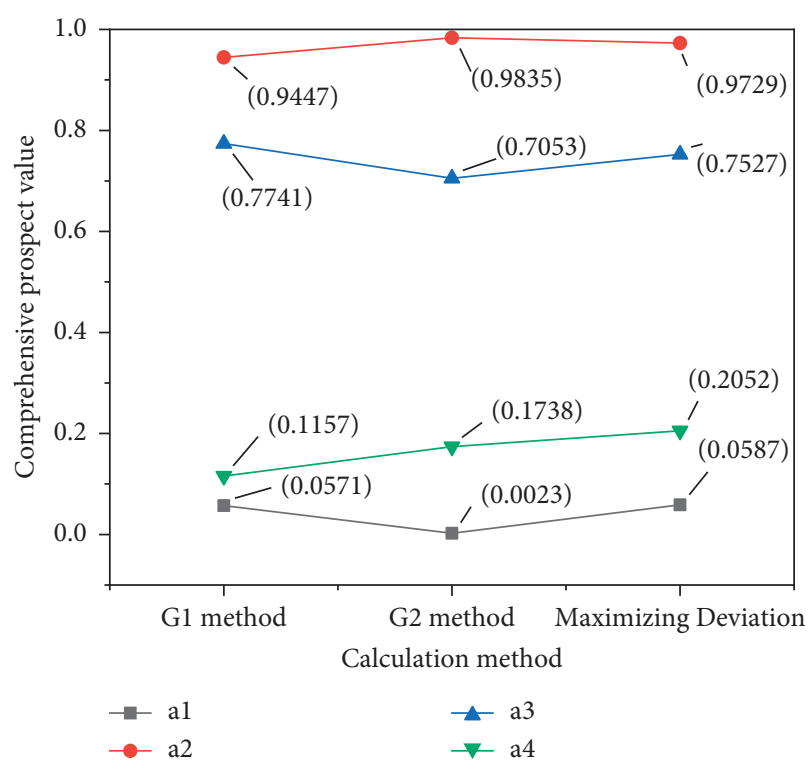

FIGURE 6: Schematic diagram of comprehensive prospect value and ranking results of schemes when $\lambda=0.9$.

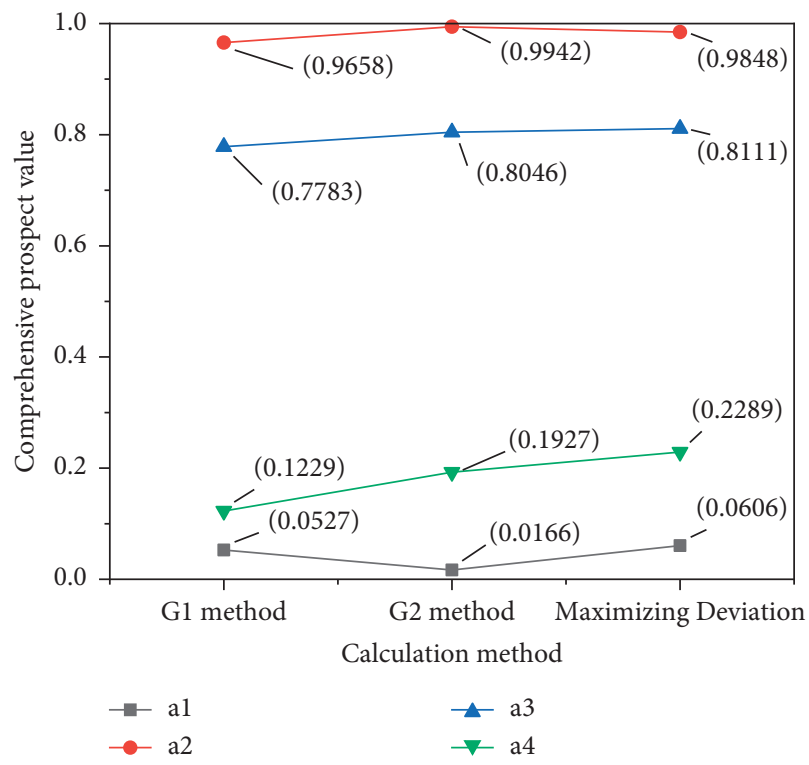

FIGURE 7: Schematic diagram of comprehensive prospect value and ranking results of schemes when $\lambda=1$.

$\lambda$ changes, the final result obtained by the method is consistent with Figure 8.

The following results can be obtained from the above discussion. When the reference point is a positive ideal scheme, the decision-maker, with the sense of loss, prefers risk. It is necessary to make $\lambda>0.5$. When the reference point is a negative ideal scheme, the decision-maker, in the profit state, dislikes risk. It is essential to make $\lambda<0.5$. That is, no matter how much $\lambda$ value is taken, when the decision-maker attaches importance to risk, it is essential to make $\lambda>0.5$; when the decision-maker belittles the risk, it is necessary to make $\lambda<0.5$. The results exert no effect on the selection of the scheme. 


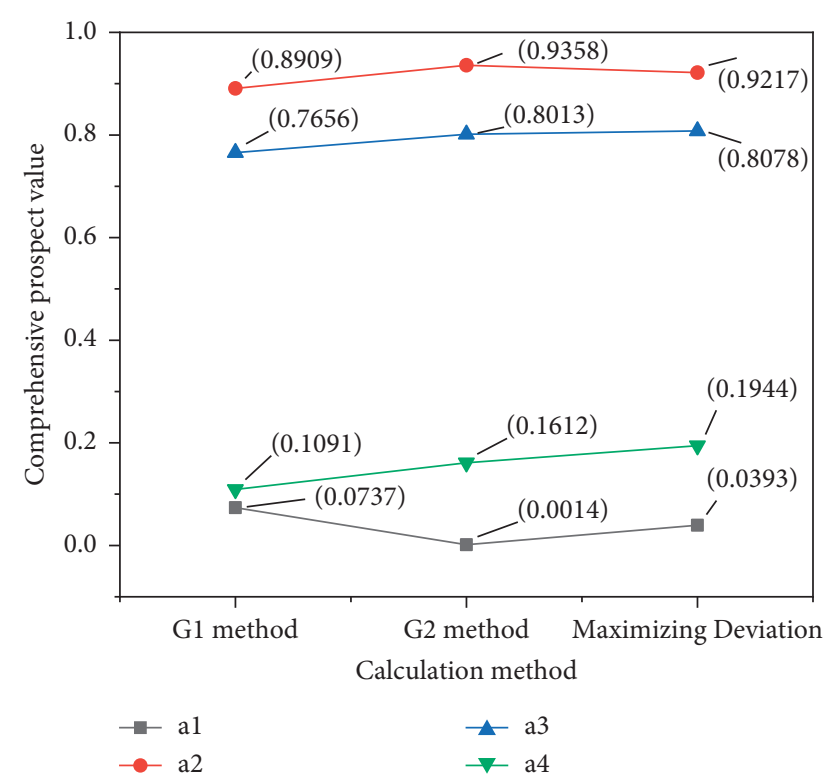

FIGURE 8: Schematic diagram of comprehensive prospect value and scheme ranking results when $\lambda=0.6$ and $\lambda=0.1$.

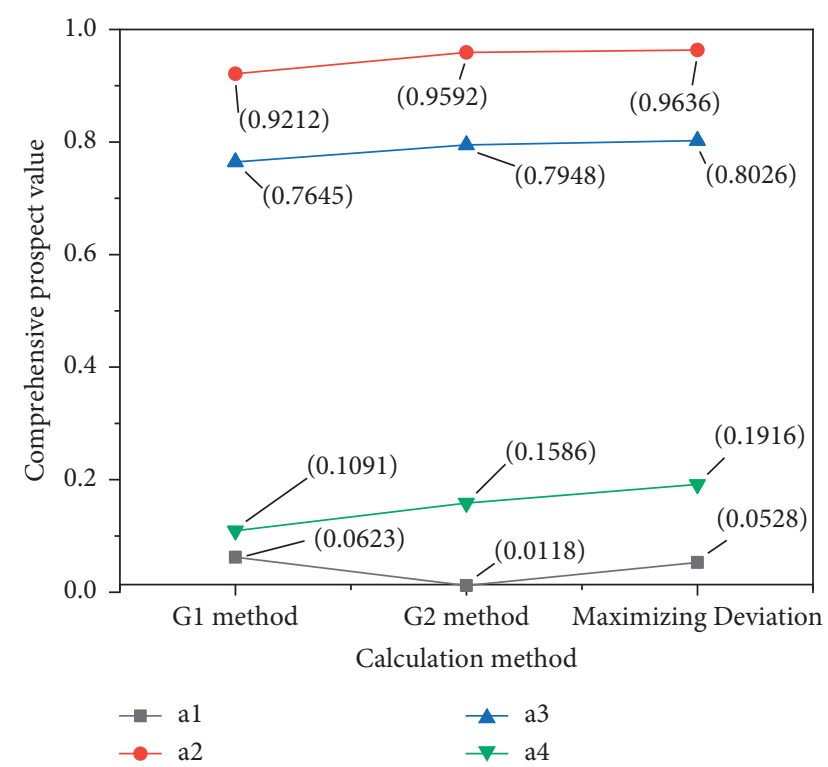

FIGURE 9: Schematic diagram of comprehensive prospect value and scheme ranking results when $\lambda=0.7$ and $\lambda=0.4$.

3.4. Results Discussion. When $G_{1}$ method, $G_{2}$ method, and deviation maximization method are employed to determine the weight of scheme criteria, although the weight is different, the ranking results of the final schemes are consistent without much difference. Therefore, a weight calculation method can be selected for subsequent enterprise credit risk management. The scheme obtained by objective method finally needs to be decided under subjective factors, so this exploration goes deep into the attitude factors of scheme decision-makers. Regarding the research content, the attitude of decision-makers is to affect the comprehensive prospect value, so as to exert an impact on the decision-making scheme. Under the four

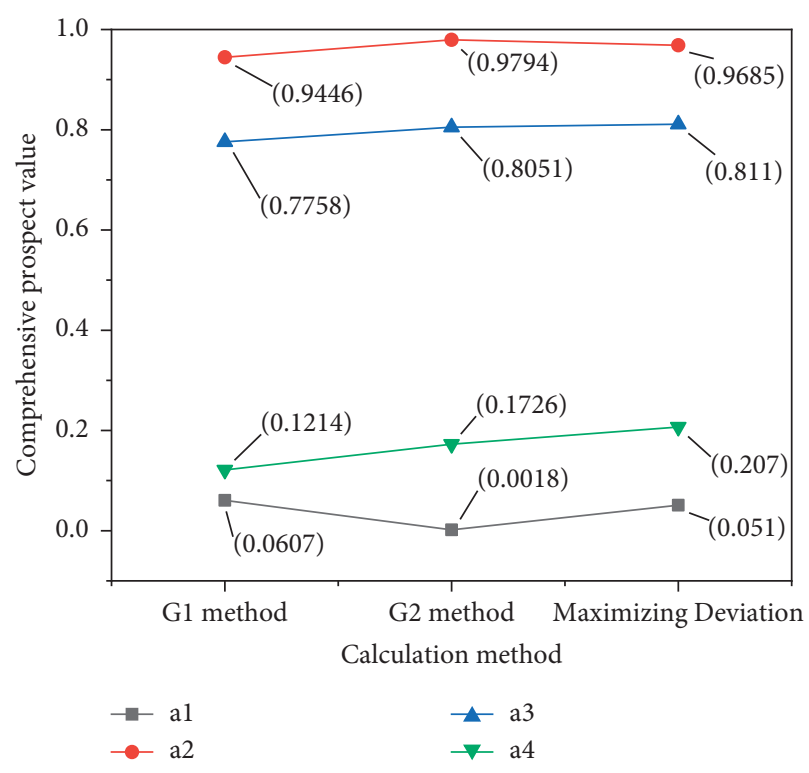

FIGURE 10: Schematic diagram of comprehensive prospect value and scheme ranking results when $\lambda=0.9$ and $\lambda=0.2$.

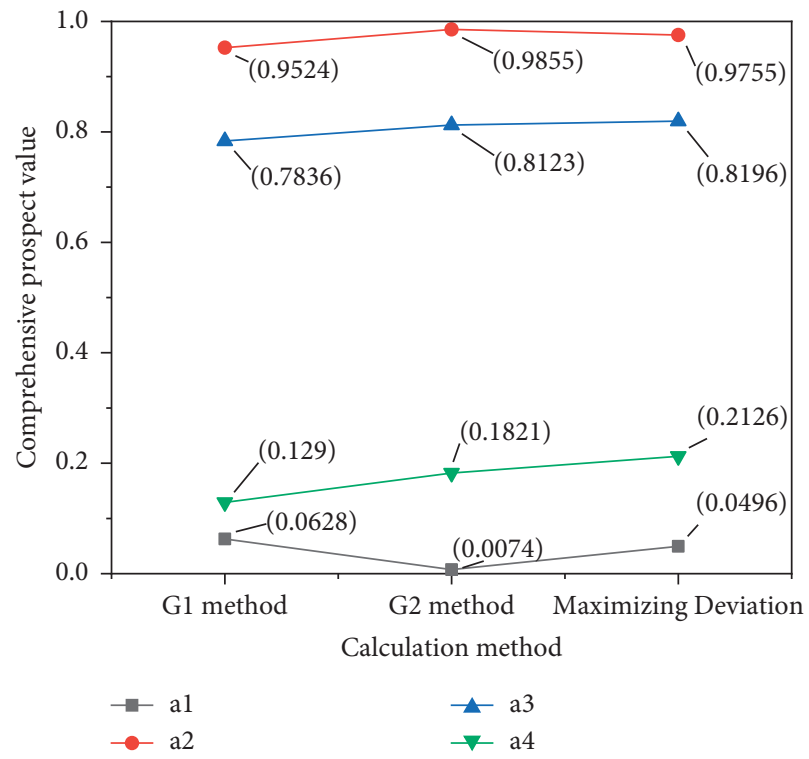

FIGURE 11: Schematic diagram of comprehensive prospect value and scheme ranking results when $\lambda=1$ and $\lambda=0$.

schemes and six criteria of this exploration, when the reference point is a positive ideal scheme, the decision-makers will have the awareness of possible loss and tend to consider the risk management. If $\lambda>0.5$, the decision can be more accurate. When the reference point is a negative ideal scheme, the decision-maker is positive to profit and has low awareness of risk management. At this time, $\lambda<0.5$ can reduce the decision-making errors of subjective factors.

\section{Conclusion}

The MCDA problem of fuzzy criteria weight in enterprise credit risk management is studied according to the prospect 
theory and fuzzy set theory. $G_{1}$ method, $G_{2}$ method, and maximizing deviation method are employed to calculate the proportion of importance degree. Meanwhile, the enterprise decision-makers' attitude towards risk is analyzed quantitatively, and whether the dynamic risk attitude value can interfere with the final decision-making is considered. The results show that it is essential to make the decision-maker's risk attitude value more than 0.5 when the degree of risk attention is high; it is essential to make the decision-maker's risk attitude value less than 0.5 when the degree of risk attention is low. Under the condition of meeting the requirements, the change of risk attitude does not influence the decision-making of the final scheme, which provides great help for the decision-makers of enterprise credit risk management facing the multischeme MCDA problem at the present stage. It can not only sort various schemes of enterprise credit risk management but also avoid the influence of decision-maker's subjective risk emotion and attitude on the final decision-making. This exploration has made great contributions to the enterprise's own credit risk management, the third-party capital market with enterprise credit risk as the enterprise assessment index, and even to the economic supervision department's prevention of systematic credit risk. It has strong practical value, but there are also shortcomings. With the development of enterprise credit risk management mode, the criteria and schemes that affect decision-making are constantly updated and iterated, which leads to the timeliness of the schemes and criteria studied. Hence, enterprise decision-makers need to be treated differently when facing new risk management problems. As a researcher in the field of credit risk management, it is expected that the enterprise credit risk management model can keep pace with the times. Moreover, more and more intelligent enterprise credit risk management decision-making methods and even advanced systems can be developed and applied.

\section{Data Availability}

The data used to support the findings of this study are available from the corresponding author upon request.

\section{Conflicts of Interest}

The author declares that there are no conflicts of interest.

\section{Acknowledgments}

This work was supported by the popularization and application of the Dual-high construction project research fruit of Henan Polytechnic "Creation Center of Contemporary Logistics Coordination” (No. 040200001).

\section{References}

[1] X. L. Li, "Talking about enterprise credit risk management," Economic Management Space, vol. 22, no. 7, pp. 32-33, 2016.

[2] B. L. Wang, "Corporate credit risk management promotes the modernization of national governance capabilities," Management Think Tank, vol. 26, no. 5, pp. 36-40, 2020.
[3] Q. Xiao, "Research on the application of financial products in corporate credit risk management," Chief Accountant of China, vol. 94, no. 9, pp. 132-133, 2019.

[4] W. Han, H. Xie, and W. C. Duan, "New method of risky multiple attribute decision making based on criteria dependence," Computer Engineering and Applications, vol. 53, no. 7, pp. 9-14, 2017.

[5] R. Zhang, Z. Xu, and X. Gou, "An integrated method for multi-criteria decision-making based on the best-worst method and Dempster-Shafer evidence theory under double hierarchy hesitant fuzzy linguistic environment," Applied Intelligence, vol. 51, no. 2, pp. 713-735, 2021.

[6] Q. Ma, K. Li, and J. Hu, "Research on personal credit evaluation based on multi-model combination," World Scientific Research Journal, vol. 5, no. 11, pp. 129-144, 2019.

[7] I. Mohammadfam, M. Mirzaei Aliabadi, A. R. Soltanian, and M. Mahdinia, "Modeling the causes-effect relationships among major accident predictors based on a fuzzy multicriteria decision-making method," Work, vol. 67, no. 2, pp. 313-321, 2020.

[8] H. D. Zhuang and X. Deng, "Research on risk-based multicriteria decision weights with incomplete information based on prospect theory," Mathematical Practice and Understanding, vol. 50, no. 24, pp. 1-8, 2020.

[9] J. Ye, J. Zhan, and Z. Xu, "A novel multi-attribute decisionmaking method based on fuzzy rough sets," Computers \& Industrial Engineering, vol. 155, no. 1, pp. 107-136, 2021.

[10] N. P. Lan, "A risk-based multi-criteria decision analysis approach to evaluating transboundary water development-the case of lower mekong river basin," Journal of Environmental Protection, vol. 12, no. 5, pp. 345-370, 2021.

[11] M. Mokarram, M. Shafie-Khah, and J. Aghaei, "Risk-based multi-criteria decision analysis of gas power plants placement in semi-arid regions," Energy Report, vol. 7, no. 2, pp. 3362-3372, 2021.

[12] M. Sahabuddin and I. Khan, "Multi-criteria decision analysis methods for energy sector's sustainability assessment: robustness analysis through criteria weight change," Sustainable Energy Technologies and Assessments, vol. 47, no. 101380, pp. 1-12, 2021.

[13] A. Pc, B. Sk, C. Cn, M. Hasan, and W. Shoombuatong, "iAMY-SCM: improved prediction and analysis of amyloid proteins using a scoring card method with propensity scores of dipeptides," Genomics, vol. 113, no. 1, pp. 689-698, 2021.

[14] M. H. Karim and M. Mohammadghasemi, "Priority determination in water resources allocation in hirmand river area," Acta Universitatis Agriculturae et Silviculturae Mendelianae Brunensis, vol. 69, no. 1, pp. 51-58, 2021.

[15] M. V. Egortsev, S. A. K. Diane, and N. D. Kaz, "Algorithmic support of the system of external observation and routing of autonomous mobile robots," Russian Technological Journal, vol. 9, no. 3, pp. 15-23, 2021.

[16] Y. Tan and W. Tang, "Guidance strategy for UAV tracking target based on reference point guidance method," Xibei Gongye Daxue Xuebao/Journal of Northwestern Polytechnical University, vol. 38, no. 1, pp. 176-182, 2020.

[17] C. Lu, M. Zhao, I. Khan, and P. Uthansakul, "Prospect theory based hesitant fuzzy multi-criteria decision making for low sulphur fuel of maritime transportation," Computers, Materials \& Continua, vol. 66, no. 2, pp. 1151-1528, 2021.

[18] N. Pramanik, N. Baidya, and N. Dhang, "Reliability assessment of three-dimensional bearing capacity of shallow foundation using fuzzy set theory," Frontiers of Structural and Civil Engineering, vol. 15, no. 2, pp. 478-489, 2021. 
[19] A. Mohtashami, "A novel modified fuzzy best-worst multicriteria decision-making method," Expert Systems with Applications, vol. 181, no. 14, Article ID 115196, 2021.

[20] X. Wang, "Multi-attribute decision-making based on new scoring function of pythagorean trapezoidal fuzzy number," Operations Research and Fuzziology, vol. 11, no. 1, pp. 63-69, 2021.

[21] M. Jin, J. Zhang, S. Cui et al., "Research on comprehensive evaluation of data link based on G1 method and entropy weight method," Journal of Physics: Conference Series, vol. 1820, no. 1, Article ID 012115, 2021.

[22] H. Yu and X. L. Yu, "A new simultaneous extension method for B-spline curves blending with G2-continuity," International Journal of Computing Science and Mathematics, vol. 11, no. 4, Article ID 385, 2020.

[23] D. Zhao, G. Wang, J. Hui, W. Hou, and R. D. Evans, "A precision analysis method for the kinematic assembly of complex products based on equivalence of deviation source," Assembly Automation, vol. 40, no. 3, pp. 447-460, 2020.

[24] L. Gao and J. Xiao, "Big data credit report in credit risk management of consumer finance," Wireless Communications and Mobile Computing, vol. 2021, no. 4, 7 pages, Article ID 4811086, 2021.

[25] B. L. Wang, "Corporate credit risk management promotes the modernization of national governance capabilities," China Insurance, vol. 2020, no. 5, 40 pages, 2020.

[26] S. Zhan and P. Wang, "Study on the credit risk management of small and medium-sized enterprises under the supply chain finance model," Shang xun, vol. 2019, no. 13, pp. 42-43, 2019.

[27] J. Guo, "Credit risk management and control in a maritime supply chain trade network," Journal of Coastal Research, vol. 106, no. 1, Article ID 109, 2020. 